\title{
Expanding with Air: Proceed with Caution
}

\author{
Chirag Shah, MD \\ Department of Radiation Oncology, Taussig Cancer Institute, Cleveland Clinic, Cleveland, OH
}

Breast cancer represents the most common noncutaneous cancer in women, with over 250,000 new cases in the USA each year. ${ }^{1}$ Breast-conserving surgery and mastectomy are both considered appropriate options for many patients; however, over the past decade, increasing rates of mastectomy have been noted. ${ }^{2,3}$ Paired with this increase in mastectomies is an increasing rate of breast reconstruction; ${ }^{3,4}$ this is important, as reconstruction represents an invaluable part of breast cancer surgery and treatment, with data consistently demonstrating improvements in quality of life and cosmetic satisfaction with reconstruction. ${ }^{5}$

Some patients undergoing mastectomy and reconstruction will require postmastectomy radiation therapy (PMRT), with benefits of PMRT including reduced locoregional recurrence and improved survival. ${ }^{6,7}$ As such, PMRT is part of the standard treatment paradigm for patients with indications and should not be omitted, due to the associated benefit. However, radiation therapy has been found to increase complications associated with breast reconstruction regardless of technique and beyond toxicities, can be associated with increased rates of revision and reconstruction loss. ${ }^{8-10}$ At this time, multiple reconstruction techniques for patients requiring postmastectomy radiation are available, including tissue expander followed by replacement with permanent implant, direct implant, or autologous reconstruction. More recently, use of air in tissue expanders (rather than saline) or self-expanding expanders filled with $\mathrm{CO}_{2}$ has begun to be incorporated into clinical practice; initial clinical data have been promising, and prospective data have demonstrated the safety and feasibility of the technique. ${ }^{1-14}$

(C) Society of Surgical Oncology 2018

First Received: 25 August 2018;

Published Online: 11 October 2018

C. Shah, MD

e-mail: csshah27@hotmail.com
However, there is a paucity of data documenting the safety and efficacy of such reconstructions in patients undergoing radiation therapy. ${ }^{14-16}$ Why is this significant? Air has very different properties compared with saline, which is similar to tissue with respect to radiation interactions. As such, this change in reconstruction technique directly impacts radiation planning and delivery, with the potential for hot and cold radiation spots. This could lead to possible increases in toxicity or underdosing, which could increase risk of recurrence. Additionally, for self-expanding devices, the metal artifact associated with the device can lead to dosimetric challenges; this is why patients with a hip replacement undergoing prostate radiation have radiation fields that avoid the replacement. However, in the case of self-expanding expanders, that is not possible, as this represents part of the treatment target. Finally, the artifact from these devices makes it difficult to delineate structures such as the internal mammary nodes; if these cannot be accurately delineated, this could increase toxicity (due to larger volumes treated based on the uncertainty of target delineation) or reduced efficacy (inability to treat key targets).

It should be noted that use of air does offer patients benefits and as such should not be abandoned for patients requiring radiotherapy, but rather further studied. ${ }^{12,13}$ What is needed before routine incorporation of expanders with air for patients requiring postmastectomy radiation therapy? First, high-quality prospective data evaluating the use of these expanders in patients undergoing radiation therapy are required, evaluating radiation planning and dose to targets as well as critical structures. This should be paired with acute, subacute, and chronic (at least 1 year posttreatment) toxicity data to provide clinicians with a toxicity profile with appropriate follow-up. Additionally, data comparing outcomes with those seen with traditional expanders or implants should be obtained, including dose comparisons as well as clinical outcomes. With such large numbers of patients undergoing mastectomy, these studies 
should be prospective and have similar patient numbers to the initial studies that evaluated the technique initially. ${ }^{14}$ This is essential as it is important to realize the significant differences between standard saline-filled and air-filled expanders; as such, using data from traditional expanders to justify the use of air expansion is not appropriate. In cases where a self-expanding expander is used, validated algorithms to account for the metal within the expander must be performed and reviewed by a group of independent radiation physicists to ensure safety. Finally, cost studies should be performed to ensure that incorporation of these techniques is cost-effective; this is likely not an issue for standard expanders filled with air, but may be with new self-expanding expanders.

How should clinicians proceed in the interim? First, in patients requiring postmastectomy radiotherapy with traditional expanders filled with air, the air should be replaced with saline. Similarly, for patients with self-expanding expanders, these should be replaced with traditional salinefilled expanders or implants; while this represents an additional procedure, until data are available for patients undergoing radiotherapy documenting safety and efficacy, this should be considered. With respect to preoperative decision-making, a multidisciplinary discussion should take place with the breast surgeon, plastic surgeon, and radiation oncologist to review the need for postmastectomy radiation and the risk of needing radiation for patients who do not have up-front indications; risk calculators can be used, for example, to evaluate the risk of nodal metastases, often an indication for treatment. ${ }^{17}$ The most conservative approach is to reserve air/ $/ \mathrm{CO}_{2}$-filled expanders for those with the lowest risk of needing radiotherapy, including those undergoing prophylactic mastectomy or mastectomy for ductal carcinoma in situ (DCIS). All other patients should not be considered for such expanders with such an approach.

While data regarding the use of air in expanders continue to grow, their use in patients requiring radiotherapy remains unsupported. As such, until further data are available to document the safety and efficacy as well as comparable outcomes to traditional expanders/implants, clinicians should proceed with caution.

DISCLOSURE Chirag Shah is a consultant for Impedimed Inc. and received research grants from Varian Medical Systems and VisionRT.

\section{REFERENCES}

1. Siegel RL, Miller KD, Jemal A. Cancer statistics, 2018. $C A$ Cancer J Clin. 2018;68:7-30.
2. Kummerow KL, Du L, Penson DF, et al. Nationwide trends in mastectomy for early-stage breast cancer. JAMA Surg. 2015;150:9-16.

3. Dragun AE, Pan J, Riley EC, et al. Increasing use of elective mastectomy and contralateral prophylactic surgery amongst breast conservation candidates: a 14-year report from a comprehensive cancer center. Am J Clin Oncol. 2013;36:375-80.

4. Cemal Y, Albomoz CR, Disa JJ et al. A paradigm shift in U.S. breast reconstruction: Part 2. The influence of changing mastectomy patterns on reconstructive rate and method. Plast Reconstr Surg. 2013;131:320e-6e.

5. Jagsi R, Li Y, Morrow M, et al. Patient-reported quality of life and satisfaction with cosmetic outcomes after breast conservation and mastectomy with and without reconstruction: results of a survey of breast cancer survivors. Ann Surg. 2015;261:1198-1206.

6. Danish Breast Cooperative Group. Study of failure pattern among high-risk breast cancer patients with or without postmastectomy radiotherapy in addition to adjuvant systemic therapy: long-term results from the Danish Breast Cancer Cooperative Group DBCG 92 b and c randomized studies. J Clin Oncol. 2006;24:2268-75.

7. Ragaz J, Olivotto IA, Spinelli JJ, et al. Locoregional radiation therapy in patients with high-risk breast cancer receiving adjuvant chemotherapy: 20-year results of the British Columbia randomized trial. J Natl Cancer Inst. 2005;97:116-26.

8. Berry $\mathrm{T}$, Brooks S, Sydow N, et al. Complications rates of radiation tissue expander and autologous tissue breast reconstruction. Ann Surg Oncol. 2010;17:202-10.

9. Ho A, Cordeiro P, Disa J, et al. Long-term outcomes in breast cancer patients undergoing 2-stage expander/implant reconstruction and postmastectomy radiation. Cancer. 2012;118:2552-9.

10. Anavekar NS, Rozen WM, Le Roux CM, et al. Achieving autologous breast reconstruction for breast cancer patients in the setting of post-mastectomy radiotherapy. $J$ Cancer Surviv. 2011;5:1-7.

11. Connell AF. Patient-activated controlled expansion for breast reconstruction with controlled carbon dioxide inflation: a feasibility study. Plast Reconstr Surg. 2011;128:848-52.

12. Connell, TF. Results from the ASPIRE study for breast reconstruction utilizing the AeroForm ${ }^{\mathrm{TM}}$ patient controlled carbon dioxide-inflated tissue expanders. J Plast Reconstr Aesthet Surg. 2015;68:1255-61.

13. Ascherman JA, Zeidler K, Morrison KA, et al. Carbon dioxidebased versus saline tissue expander for breast reconstruction: results of the XPAND prospective, randomized clinical trial. Plast Reconstr Surg. 2016;138:1161-70.

14. Rembert JL, Heitz R, Hoffman A. Radiation testing of the AeroForm $\mathrm{CO}_{2}$-based breast tissue expander implant. Radiat Oncol. 2013;8;235.

15. Tran T, Ding W, Subramania B, et al. A dosimetric analysis of the Aeroform ${ }^{\mathrm{TM}}$ tissue expander in radiation therapy. Int $J$ Cancer Ther Oncol. 2014;2:1-6.

16. Moni J, Saleeby J, Bannon E, et al. Dosimetric impact of the AeroForm tissue expander in postmastectomy radiation therapy: an ex vivo analysis. Pract Radiat Oncol. 2014;5:e1-e8.

17. Memorial Sloan Kettering Cancer Center. Breast Cancer Nomogram: Sentinel Lymph Node Metastasis. Available at: http:// nomograms.mskcc.org/breast/BreastSLNodeMetastasisPage.aspx. Accessed August 25, 2018. 